\title{
How to construct good measures ${ }^{1}$
}

\author{
Augusto C. Ponce
}

Dedicated to H. Brezis in the occasion of his 60th birthday

November 13, 2004

Abstract. Given any continuous nondecreasing function $g: \mathbb{R} \rightarrow \mathbb{R}$, with $g(t)=0$, $\forall t \leq 0$, we show that there always exists some positive measure $\mu$, concentrated on a set of zero Newtonian capacity, for which the problem

$$
\left\{\begin{aligned}
-\Delta u+g(u) & =\mu & & \text { in } \Omega, \\
u & =0 & & \text { on } \partial \Omega,
\end{aligned}\right.
$$

admits a solution. This provides an affirmative answer to Open problem 2 raised by Brezis-Marcus-Ponce [3]. When $N \geq 3$ and $g(t)=\mathrm{e}^{t}-1, \forall t \geq 0$, Bartolucci-LeoniOrsina-Ponce [1] proved that any measure $\mu \leq 4 \pi \mathcal{H}^{N-2}$ is good for problem (0.1). We present examples of other good measures which are not $\leq 4 \pi \mathcal{H}^{N-2}$.

Mathematics Subject Classification (2000). 35J60, 35B05.

Key words. Nonlinear elliptic equations, good measures, Cantor sets.

\section{Introduction}

Let $\Omega \subset \mathbb{R}^{N}, N \geq 2$, be a smooth bounded domain. Let $g: \mathbb{R} \rightarrow \mathbb{R}$ be a continuous nondecreasing function such that $g(t)=0, \forall t \leq 0$. Given a bounded measure $\mu \in \mathcal{M}(\Omega)$, then $u$ is a solution of

$$
\left\{\begin{aligned}
-\Delta u+g(u) & =\mu & & \text { in } \Omega \\
u & =0 & & \text { on } \partial \Omega
\end{aligned}\right.
$$

if $u \in L^{1}(\Omega), g(u) \in L^{1}(\Omega)$, and

$$
-\int_{\Omega} u \Delta \zeta+\int_{\Omega} g(u) \zeta=\int_{\Omega} \zeta d \mu \quad \forall \zeta \in C^{2}(\bar{\Omega}), \zeta=0 \text { on } \partial \Omega .
$$

We say that $\mu$ is a good measure (relative to $g$ ) if (1.1) has a solution $u$. We observe that $u$, whenever it exists, is unique. The study of problem (1.1), when $\mu \in L^{1}(\Omega)$, was initiated by Brezis-Strauss [5]. They established that every measure in $L^{1}(\Omega)$ is good. Later, Bénilan-Brezis [2] (see also Brezis-Véron [6])

\footnotetext{
${ }^{1}$ Proceedings of the Fifth European Conference on Elliptic and Parabolic Problems. A special tribute to the work of Haïm Brezis.
} 
proved that (1.1) need not have a solution for a given measure $\mu$. In fact, if $N \geq 3$ and $g(t)=t^{p}, \forall t \geq 0$, for some $p \geq \frac{N}{N-2}$, then there exists no $u$ satisfying (1.1) for $\mu=\delta_{a}, a \in \Omega$.

Let $\mathcal{G}(g)$ denote the set of good measures associated to $g$. One can show (see [3]) that $\mathcal{G}(g)$ is convex and closed with respect to the strong topology in $\mathcal{M}(\Omega)$.

A measure $\mu$ is diffuse if $\mu(A)=0$ for every Borel set $A \subset \Omega$ such that $\operatorname{cap}(A)=0$, where "cap" denotes the Newtonian $\left(H^{1}\right)$ capacity. If $\mu \in \mathcal{M}(\Omega)$ and $\mu^{+}$is diffuse, then $\mu$ is good for every nonlinearity $g$ (see [3, Corollary 3$]$ ). The converse is also true. Namely, if $\mu$ is good for every $g$, then $\mu^{+}$is diffuse (see $[3$, Theorem 5]). We can summarize this as

$$
\left\{\mu \in \mathcal{M}(\Omega): \mu^{+} \text {is a diffuse measure }\right\}=\bigcap_{g} \mathcal{G}(g),
$$

where the intersection is taken over all continuous nondecreasing functions $g: \mathbb{R} \rightarrow$ $\mathbb{R}$ such that $g(t)=0, \forall t \leq 0$.

One of our main results is the following

Theorem 1 Given any $g$, we have

$$
\left\{\mu \in \mathcal{M}(\Omega): \mu^{+} \text {is a diffuse measure }\right\} \varsubsetneqq \mathcal{G}(g) .
$$

In other words, for any fixed $g$, there exists a measure $\mu \in \mathcal{M}(\Omega), \mu \geq 0$, such that $\mu \in \mathcal{G}(g)$, but $\mu$ is not diffuse.

Theorem 1 gives a positive answer to Open problem 2 in [3]. As we shall see below, the proof of Theorem 1 is constructive. In fact, it gives a recipe for explicitly obtaining the measure $\mu$. Of course, such $\mu$ will heavily depend on the function $g$.

In dimension $N \geq 3$, Theorem 1 can be improved. Recall that any Borel set $A \subset \Omega$ such that $\mathcal{H}^{N-2}(A)<\infty$ has zero capacity (but the converse is false; see [7]). When $N \geq 3$, it is always possible to find good measures $\mu$ of the form $\mu=\alpha \mathcal{H}^{N-2}\left\lfloor_{K}\right.$ for some compact set $K \subset \Omega$ and $\alpha>0$. More precisely, we have

Theorem 2 Assume $N \geq 3$. Given any $g$, there exists a compact set $K \subset \Omega$, $\mathcal{H}^{N-2}(K) \in(0, \infty)$, such that $\mu=\alpha \mathcal{H}^{N-2}\left\lfloor_{K}\right.$ is good (relative to $g$ ) for every $\alpha>0$.

Theorem 2 is no longer true in dimension $N=2$. In fact, problem (1.1) has no solution when $g(t)=\mathrm{e}^{t}-1, \forall t \geq 0$, and $\mu=\alpha \delta_{a}, a \in \Omega$, for any $\alpha>4 \pi$ (see Vázquez [12]).

One can also construct good measures $\mu \geq 0$ concentrated on a set of zero $\mathcal{H}^{N-2}$-measure. In fact,

Theorem 3 Assume $N \geq 3$. For any $g$, there exists a good measure $\mu \geq 0$ such that $\mathcal{H}^{N-2}(\operatorname{supp} \mu)=0$. 
When $N \geq 3$ and $g(t)=\mathrm{e}^{t}-1, \forall t \geq 0$, it has been established in [1] that if $\mu \leq 4 \pi \mathcal{H}^{N-2}$, then $\mu$ is good. According to Theorems 2 and 3 above, there are other good measures which are not $\leq 4 \pi \mathcal{H}^{N-2}$. The existence of such measures was suggested by L. Véron in a personal communication.

The construction presented here has been applied in the study of other related problems; see [4] and [8]. An alternative approach for obtaining good measures which are not diffuse might be found in some recent work of Marcus-Véron [10].

This paper is organized as follows. In Section 2, we define a Cantor-type set $F$ associated to a subsequence $\left(\ell_{k_{j}}\right)$; as we shall see later on, the proofs of Theorems 1-3 rely on suitable choices of $\left(\ell_{k}\right)$ and $\left(k_{j}\right)$. We then introduce a positive measure $\mu_{F}$ supported on $F$. In Section 3 , we estimate the potential generated by $\mu_{F}$ in terms of $\left(\ell_{k_{j}}\right)$. In Section 4 , we present the proofs of Theorems 2 and 3; as a corollary, we obtain Theorem 1 when $N \geq 3$. Finally, in Section 5 , we prove Theorem 1 in the case $N=2$.

\section{Construction of the Cantor set $F$ associated to the subsequence $\left(\ell_{k_{j}}\right)$}

We shall assume for simplicity that $\Omega=Q_{1}$, the unit cube centered at 0 . One of the main ingredients in the proofs of Theorems 1-3 will be the construction of a (generalized) Cantor set $F \subset \Omega$; see e.g. [11]. We begin by describing the building blocks used in the definition of $F$.

Let $n \geq 1$ be an integer and let $0<s \ll t$. We shall associate to the triple $(s, t, n)$ a compact set $E(s, t, n) \subset\left[-\frac{t}{2}, \frac{t}{2}\right]^{N}$ in the following way. Let

$$
\alpha=\frac{t-n s}{n-1} \text {. }
$$

For $j=1, \ldots, n$, set

$$
a_{j}=(j-1)(s+\alpha)-\frac{t}{2} \quad \text { and } \quad b_{j}=a_{j}+s .
$$

In particular, $a_{1}=-\frac{t}{2}$ and $b_{n}=\frac{t}{2}$. We then define

$$
E(s, t, n)=\bigcup_{1 \leq i_{1}, \ldots, i_{N} \leq n}\left[a_{i_{1}}, b_{i_{1}}\right] \times \cdots \times\left[a_{i_{N}}, b_{i_{N}}\right] .
$$

Thus, the set $E(s, t, n)$ is the union of $n^{N}$ cubes of side $s$, uniformly distributed in $\left[-\frac{t}{2}, \frac{t}{2}\right]^{N}$. The distance between two components of $E(s, t, n)$ is $\geq \alpha$.

We now turn to the construction of $F$.

Let $\left(\ell_{k}\right)$ be a decreasing sequence of positive numbers such that

$$
\ell_{1} \leq \frac{1}{4} \quad \text { and } \quad \ell_{k+1} \leq \theta \ell_{k} \quad \forall k \geq 1
$$


for some $\theta \in\left(0, \frac{1}{2}\right)$. The Cantor set $F$ associated to the subsequence $\left(\ell_{k_{j}}\right)$ is defined by induction as follows.

Let $F_{0}=Q_{1}, k_{0}=0$ and $\ell_{0}=1$. Let $F_{j}$ be the set obtained after the $j$-th step; $F_{j}$ is the disjoint union of $2^{N k_{j}}$ cubes $Q_{i}$ of side $\ell_{k_{j}}$. Let $x_{1}, \ldots, x_{2^{N k_{j}}}$ denote the centers of each component of $F_{j}$ (although it is not indicated, such points do depend on $j$ ). We then set

$$
F_{j+1}=\bigcup_{i=1}^{2^{N k_{j}}} E\left(\ell_{k_{j+1}}, \gamma \ell_{k_{j}}, 2^{\left(k_{j+1}-k_{j}\right)}\right)+x_{i}
$$

where $\gamma=\frac{1}{2}+\theta \in\left(\frac{1}{2}, 1\right)$. In particular, $F_{j+1}$ is the union of $2^{N k_{j+1}}$ disjoint cubes of side $\ell_{k_{j+1}}$. Moreover, since we are taking $t=\gamma \ell_{k_{j}}$, we have

$$
d\left(F_{j+1}, \partial F_{j}\right)=\frac{1-\gamma}{2} \ell_{k_{j}}=\frac{1-2 \theta}{4} \ell_{k_{j}}
$$

We also point out that the distance between any two components of $F_{j+1}$ inside the cube $\left[-\gamma \ell_{k_{j}}, \gamma \ell_{k_{j}}\right]^{N}+x_{i}$ is $\geq \alpha$, where $\alpha$ is given by (2.1). Since (2.2) holds with $\theta<\frac{1}{2}$, we have

$$
\alpha \sim \frac{\ell_{k_{j}}}{2^{\left(k_{j+1}-k_{j}\right)}} .
$$

We finally set

$$
F=\bigcap_{j=0}^{\infty} F_{j}
$$

We would like to emphasize the main feature in the construction of $F$. In order to obtain a standard Cantor set, inside each component $Q_{i}$ of $F_{j}$ one would take $2^{N}$ small cubes. In our case, we select $2^{N\left(k_{j+1}-k_{j}\right)}$ small cubes inside $Q_{i}$. This possibility of choosing many more cubes turns out to be crucial in the proofs of some of our main results.

\section{Potential generated by the uniform measure $\mu_{F}$ concentrated on $\boldsymbol{F}$}

In this section, we present some basic estimates which will be used throughout this paper.

For each $j \geq 1$, let $\mu_{j}=\frac{1}{\left|F_{j+1}\right|} \chi_{F_{j+1}}$, where $F_{j+1}$ is given by (2.3). The uniform measure concentrated on $F, \mu_{F}$, is the weak* limit of $\left(\mu_{j}\right)$ in $\mathcal{M}(\Omega)$ as $j \rightarrow \infty$. In particular, $\mu_{F} \geq 0$ and $\mu_{F}(\Omega)=1$. A key property satisfied by $\mu_{F}$ is given by the next 
Lemma 1 For every $x \in F_{j+1}, j \geq 0$, we have

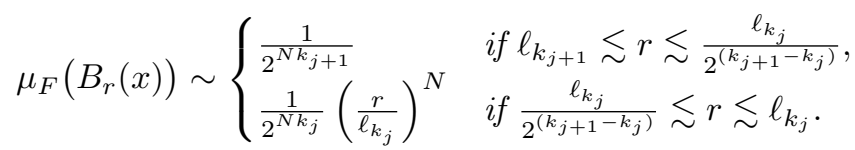

Here, we implicitly assume that $k_{0}=0$. We say that $a \lesssim b$ if there exists $C>0$, depending on $N$ and $\theta$, such that $a \leq C b$. By $a \sim b$, we mean that $a \lesssim b$ and $b \lesssim a$.

Proof. We shall use the same notation as in the construction of $F$. Note that if

$$
\ell_{k_{j+1}} \lesssim r \lesssim \frac{\ell_{k_{j}}}{2^{\left(k_{j+1}-k_{j}\right)}}
$$

then $B_{r}(x)$ contains a single component $Q_{i, n}$ of $F_{j+1}$. Since

$$
\mu_{F}\left(Q_{i, n}\right)=\frac{1}{2^{N k_{j+1}}}
$$

the first estimate in (3.1) follows.

We now assume

$$
\frac{\ell_{k_{j}}}{2^{\left(k_{j+1}-k_{j}\right)}} \lesssim r \lesssim \ell_{k_{j}}
$$

Let $Q_{i}$ be the component of $F_{j}$ containing $x$. Recall that there are $2^{N\left(k_{j+1}-k_{j}\right)}$ components $Q_{i, n}$ of $F_{j+1}$ contained in $Q_{i}$. Thus, the number of cubes $Q_{i, n}$ contained in $B_{r}(x)$ is of the order of $2^{N\left(k_{j+1}-k_{j}\right)}\left(\frac{r}{\ell_{k_{j}}}\right)^{N}$. Since, for each $Q_{i, n}$, $\mu_{F}\left(Q_{i, n}\right)=\frac{1}{2^{N k_{j+1}}}$, we then have

$$
\mu_{F}\left(B_{r}(x)\right) \sim 2^{N\left(k_{j+1}-k_{j}\right)}\left(\frac{r}{\ell_{k_{j}}}\right)^{N} \mu_{F}\left(Q_{i, n}\right)=\frac{1}{2^{N k_{j}}}\left(\frac{r}{\ell_{k_{j}}}\right)^{N} .
$$

The proof of the lemma is complete.

Let $v \in L^{1}\left(Q_{1}\right)$ be the unique solution of

$$
\left\{\begin{aligned}
-\Delta v & =\mu_{F} & & \text { in } Q_{1} \\
v & =0 & & \text { on } \partial Q_{1} .
\end{aligned}\right.
$$

A basic estimate satisfied by $v$ is given by the following

Proposition 1 Assume $N \geq 3$. Let $F \subset Q_{1}$ be the Cantor set associated to the subsequence $\left(\ell_{k_{j}}\right)$ and let $v$ be the solution of (3.2). Then, there exist constants $C_{1}, C_{2}>0$ (depending on $N$ and $\theta$ ) such that

$$
C_{1}\left(\frac{1}{\ell_{k_{1}}^{N-2}}+\sum_{i=1}^{j} \frac{1}{2^{N k_{i}} \ell_{k_{i}}^{N-2}}\right) \leq v(x) \leq C_{2}\left(\frac{1}{\ell_{k_{1}}^{N-2}}+\sum_{i=1}^{j} \frac{1}{2^{N k_{i}} \ell_{k_{i}}^{N-2}}\right),
$$

for every $x \in \partial F_{j}, j \geq 1$. 
Proof. Let

$$
w(x)=\frac{1}{N \omega_{N}} \int_{0}^{\infty} \frac{\mu_{F}\left(B_{r}(x)\right)}{r^{N-1}} d r \quad \forall x \in Q_{1},
$$

where $\omega_{N}=\left|B_{1}\right|$. By (2.4), for every $x \in \partial F_{j}$ we have

$$
\mu_{F}\left(B_{r}(x)\right)=0 \quad \text { if } r \lesssim \ell_{k_{j}},
$$

so that

$$
w(x) \sim \int_{\ell_{k_{j}}}^{\infty} \frac{\mu_{F}\left(B_{r}(x)\right)}{r^{N-1}} d r \quad \forall x \in \partial F_{j} .
$$

Thus,

$$
w(x) \sim \sum_{i=1}^{j-1} \int_{\ell_{k_{i+1}}}^{\ell_{k_{i}}} \frac{\mu_{F}\left(B_{r}(x)\right)}{r^{N-1}} d r+\int_{\ell_{k_{1}}}^{\infty} \frac{\mu_{F}\left(B_{r}(x)\right)}{r^{N-1}} d r \sim \sum_{i=1}^{j-1}\left(A_{i}+B_{i}\right)+\frac{1}{\ell_{k_{1}}^{N-2}},
$$

where, by Lemma 1 and (2.2),

$$
A_{i}=\int_{\ell_{k_{i+1}}}^{\frac{\ell_{k_{i}}^{\left(k_{i+1}-k_{i}\right)}}{2^{\prime}}} \frac{\mu_{F}\left(B_{r}(x)\right)}{r^{N-1}} d r \sim \frac{1}{2^{N k_{i+1}}} \int_{\ell_{k_{i+1}}}^{\frac{\ell_{k_{i}}^{\left(k_{i+1}-k_{i}\right)}}{r^{N-1}}} \frac{d r}{2^{N k_{i+1}} \ell_{k_{i+1}}^{N-2}}
$$

and

$$
B_{i}=\int_{\frac{\ell_{k_{i}}}{2^{\left(k_{i+1}-k_{i}\right)}}}^{\ell_{k_{i}}} \frac{\mu_{F}\left(B_{r}(x)\right)}{r^{N-1}} d r \sim \frac{1}{2^{N k_{i}} \ell_{k_{i}}^{N}} \int_{\frac{\ell^{\left(k_{i+1}-k_{i}\right)}}{\ell_{k_{i}}}}^{\ell_{k_{i}}} \quad r d r \sim \frac{1}{2^{N k_{i}} \ell_{k_{i}}^{N-2}} .
$$

Therefore,

$$
w(x) \sim \sum_{i=1}^{j-1}\left(\frac{1}{2^{N k_{i+1}} \ell_{k_{i+1}}^{N-2}}+\frac{1}{2^{N k_{i}} \ell_{k_{i}}^{N-2}}\right)+\frac{1}{\ell_{k_{1}}^{N-2}} \sim \sum_{i=1}^{j} \frac{1}{2^{N k_{i}} \ell_{k_{i}}^{N-2}}+\frac{1}{\ell_{k_{1}}^{N-2}} .
$$

In other words, $w$ satisfies (3.3). On the other hand, we have

$$
d\left(F_{1}, \partial Q_{1}\right)=\frac{1-\gamma}{2}=\frac{1-2 \theta}{4}>0 .
$$

Since $w \geq 0$ and $-\Delta w=\mu_{F}$ in $Q_{1}$ (see Lemma 2 below), there exist constants $\tilde{C}_{1}, \tilde{C}_{2}>0$ such that

$$
\tilde{C}_{1} w \leq v \leq \tilde{C}_{2} w \quad \text { on } F_{1} .
$$

Combining (3.4) and (3.5), we obtain (3.3). This concludes the proof of the proposition.

We now establish a well-known fact used in the proof of Proposition 1: 
Lemma 2 Given $\mu \in \mathcal{M}\left(\mathbb{R}^{N}\right)$, let

$$
w(x)=\frac{1}{N \omega_{N}} \int_{0}^{\infty} \frac{\mu\left(B_{r}(x)\right)}{r^{N-1}} d r \quad \forall x \in \mathbb{R}^{N} .
$$

Then,

$$
-\Delta w=\mu \quad \text { in } \mathcal{D}^{\prime}\left(\mathbb{R}^{N}\right) .
$$

Proof. We shall prove the lemma for $N \geq 3$; the case $N=2$ is similar.

We make the change of variables $r=s^{-\frac{1}{N-2}}$ in (3.6). Since $\frac{d r}{r^{N-1}}=-\frac{d s}{N-2}$, we get

$$
\begin{aligned}
N(N-2) \omega_{N} w(x) & =(N-2) \int_{0}^{\infty} \mu\left(\left\{y \in \mathbb{R}^{N}:|x-y|<r\right\}\right) \frac{d r}{r^{N-1}} \\
& =\int_{0}^{\infty} \mu\left(\left\{y \in \mathbb{R}^{N}:|x-y|<s^{-\frac{1}{N-2}}\right\}\right) d s \\
& =\int_{0}^{\infty} \mu\left(\left\{y \in \mathbb{R}^{N}: \frac{1}{|x-y|^{N-2}}>s\right\}\right) d s=\int_{\mathbb{R}^{N}} \frac{d \mu(y)}{|x-y|^{N-2}},
\end{aligned}
$$

from which the result follows.

The counterpart of Proposition 1 in dimension $N=2$ is given by

Proposition 2 Assume $N=2$. Let $F \subset Q_{1}$ be the Cantor set associated to the subsequence $\left(\ell_{k_{j}}\right)$ and let $v$ be the solution of (3.2). Then, for every $j \geq 1$, we have

$$
v \sim\left(\log \frac{1}{\ell_{k_{1}}}+\sum_{i=1}^{j} \frac{1}{4^{k_{i}}} \log \frac{1}{\ell_{k_{i}}}\right) \quad \text { on } \partial F_{j} .
$$

The proof of Proposition 2 follows along the same lines and shall be omitted.

\section{Proofs of Theorems 2 and 3}

We start by recalling the definition of the (spherical) Hausdorff measure $\mathcal{H}^{s}$ in $\mathbb{R}^{N}$, where $0 \leq s \leq N$. Let $A \subset \mathbb{R}^{N}$ be a Borel set. Given $\delta>0$, let

$$
\mathcal{H}_{\delta}^{s}(A)=\inf \left\{\sum_{i} \omega_{s} r_{i}^{s}: K \subset \bigcup_{i} B_{r_{i}} \text { with } r_{i}<\delta, \forall i\right\},
$$

where the infimum is taken over all coverings of $A$ with open balls $B_{r_{i}}$ of radii $r_{i}<\delta$, and $\omega_{s}=\frac{\pi^{s / 2}}{\Gamma\left(\frac{s}{2}+1\right)}$. When $s$ is a positive integer, then $\omega_{s}$ is the measure of the unit ball in $\mathbb{R}^{s}$. We then set

$$
\mathcal{H}^{s}(A)=\lim _{\delta \downarrow 0} \mathcal{H}_{\delta}^{s}(A) .
$$

We have the following 
Lemma 3 Let $F$ be the Cantor set associated to the subsequence $\left(\ell_{k_{j}}\right)$. Then,

$$
\mathcal{H}^{s}(F) \sim \liminf _{j \rightarrow \infty} 2^{N k_{j}} \ell_{k_{j}}^{s}
$$

Moreover, if $\mathcal{H}^{s}(F) \in(0, \infty)$, then

$$
\mu_{F}=\frac{1}{\mathcal{H}^{s}(F)} \mathcal{H}^{s}\left\lfloor_{F} .\right.
$$

\section{Proof.}

Proof of (4.1). For $j \geq 1$ fixed, let $\left(B_{i}\right)$ be a covering of $F$ with $2^{N k_{j}}$ balls of radii $\ell_{k_{j}}$, where each ball $B_{i}$ is concentric to some component of $F_{j}$. Then,

$$
\mathcal{H}_{\delta}^{s}(F) \leq \omega_{s} 2^{N k_{j}} \ell_{k_{j}}^{s}
$$

for every $\delta>\ell_{k_{j}}$. Thus,

$$
\mathcal{H}^{s}(F) \leq \omega_{s} \liminf _{j \rightarrow \infty} 2^{N k_{j}} \ell_{k_{j}}^{s}
$$

which gives $\lesssim$ in $(4.1)$.

Conversely, if $\liminf _{j \rightarrow \infty} 2^{N k_{j}} \ell_{k_{j}}^{s}=0$, then it follows from (4.3) that $\mathcal{H}^{s}(F)=0$ and we are done. We now assume that

$$
\liminf _{j \rightarrow \infty} 2^{N k_{j}} \ell_{k_{j}}^{s}>0
$$

(the limit above possibly being infinite). Given $0<a<\liminf _{j \rightarrow \infty} 2^{N k_{j}} \ell_{k_{j}}^{s}$, let $j_{0} \geq 1$ be sufficiently large so that

$$
2^{N k_{j}} \ell_{k_{j}}^{s} \geq a \quad \forall j \geq j_{0}
$$

It then follows from Lemma 1 and (4.4) that there exists $C>0$ such that

$$
\mu_{F}\left(B_{r}(x)\right) \leq \frac{C r^{s}}{a} \quad \forall x \in F, \quad \forall r \in\left(0, \ell_{j_{0}}\right) .
$$

Let $\delta \in\left(0, \ell_{j_{0}}\right)$ and let $\left(B_{r_{i}}\right)$ be a covering of $F$ with balls of radii $r_{i}<\delta$. Without loss of generality, we may assume that each $B_{r_{i}}$ is centered at some point of $F$. Thus, in view of (4.5), we have

$$
\sum_{i} r_{i}^{s} \geq \frac{a}{C} \sum_{i} \mu_{F}\left(B_{r_{i}}\right) \geq \frac{a}{C} \mu_{F}\left(\bigcup_{i} B_{r_{i}}\right)=\frac{a}{C} \mu_{F}(F)=\frac{a}{C}
$$

This lower bound holds for any covering $\left(B_{r_{i}}\right)$ such that $r_{i}<\delta, \forall i$. Therefore,

$$
\mathcal{H}^{s}(F) \geq \mathcal{H}_{\delta}^{s}(F) \geq \frac{\omega_{s}}{C} a .
$$


Since $a<\liminf _{j \rightarrow \infty} 2^{N k_{j}} \ell_{k_{j}}^{s}$ was arbitrary, we conclude that

$$
\mathcal{H}^{s}(F) \geq \frac{\omega_{s}}{C} \liminf _{j \rightarrow \infty} 2^{N k_{j}} \ell_{k_{j}}^{s}
$$

This establishes (4.1).

Proof of (4.2). Assume $\mathcal{H}^{s}(F) \in(0, \infty)$. Let $Q_{i}$ be a component of $F_{j}, j \geq 1$. By symmetry, we have

$$
\mathcal{H}^{s}(F)=2^{N k_{j}} \mathcal{H}^{s}\left(Q_{i} \cap F\right) .
$$

Since $\mu_{F}\left(Q_{i}\right)=2^{-N k_{j}}$, we get

$$
\mu_{F}\left(Q_{i}\right)=\frac{1}{\mathcal{H}^{s}(F)} \mathcal{H}^{s}{ }_{F}\left(Q_{i}\right)
$$

Given $A \subset \mathbb{R}^{N}$ open, we may write $A \cap F=\bigcup_{i}\left(Q_{i} \cap F\right)$, where $\left(Q_{i}\right)$ is a family of disjoint connected components among all $F_{j}, j \geq 1$. It then follows from (4.6) that

$$
\mu_{F}(A)=\frac{1}{\mathcal{H}^{s}(F)} \mathcal{H}^{s}\left\lfloor_{F}(A) \text { for every open set } A \subset \mathbb{R}^{N}\right.
$$

Since $\mu_{F}$ and $\mathcal{H}^{s}\left\lfloor_{F}\right.$ are Radon measures, (4.2) follows. This concludes the proof of the lemma.

We recall the following result (see [3, Theorem 4]):

Proposition 3 Suppose $\mu_{1} \in \mathcal{M}(\Omega)$ is a good measure for problem (1.1). Then, any measure $\mu_{2} \leq \mu_{1}$ is also good.

We now establish the

Proposition 4 Assume $N \geq 3$. Let $F$ be the Cantor set associated to the subsequence $\left(\ell_{k_{j}}\right)$. There exists $C>0$ (depending on $N$ and $\theta$ ) such that if

$$
\sum_{j=1}^{\infty} g\left(C \alpha_{0} \sum_{i=1}^{j+1} \frac{1}{2^{N k_{i}} \ell_{k_{i}}^{N-2}}\right) 2^{N k_{j}} \ell_{k_{j}}^{N}<\infty \quad \text { for some } \alpha_{0}>0
$$

then $\alpha_{0} \mu_{F} \in \mathcal{G}(g)$.

Proof. Let

$$
a=\frac{1}{\ell_{k_{1}}^{N-2}} \quad \text { and } \quad b_{j}=\sum_{i=1}^{j} \frac{1}{2^{N k_{i}} \ell_{k_{i}}^{N-2}} \quad \forall j \geq 1 .
$$

Let $v$ be the solution of (3.2). By Proposition 1, there exists $C_{2}>0$ such that

$$
v(x) \leq C_{2}\left(a+b_{j}\right) \quad \forall x \in \partial F_{j} .
$$


Note that $v$ is harmonic in (int $\left.F_{j}\right) \backslash F_{j+1}$. Thus, by the maximum principle,

$$
v(x) \leq C_{2}\left(a+b_{j+1}\right) \quad \forall x \in F_{j} \backslash F_{j+1} .
$$

Assume that $\lim _{j \rightarrow \infty} b_{j}<\infty$. In this case, we have $v \in L^{\infty}(\Omega)$; hence, $g\left(\alpha_{0} v\right) \in$ $L^{1}(\Omega)$. We then conclude that $\alpha_{0} \mu_{F}+g\left(\alpha_{0} v\right)$ is good. By Proposition $3, \alpha_{0} \mu_{F}$ is also a good measure.

We now assume that

$$
\lim _{j \rightarrow \infty} b_{j}=\infty
$$

Since

$$
\left|F_{j} \backslash F_{j+1}\right| \leq\left|F_{j}\right|=2^{N k_{j}} \ell_{k_{j}}^{N},
$$

then, for every $\alpha>0$, we have

$$
\begin{aligned}
\int_{\Omega} g(\alpha v) & =\sum_{j=1}^{\infty} \int_{F_{j} \backslash F_{j+1}} g(\alpha v)+\int_{\Omega \backslash F_{1}} g(\alpha v) \\
& \leq \sum_{j=1}^{\infty} g\left(C_{2} \alpha\left(a+b_{j+1}\right)\right) 2^{N k_{j}} \ell_{k_{j}}^{N}+O(1) .
\end{aligned}
$$

Using (4.8), we have $C_{2} \alpha\left(a+b_{j+1}\right) \leq 2 C_{2} \alpha b_{j+1}$ for every $j \geq 1$ sufficiently large. Therefore, if (4.7) holds with $C=2 C_{2}$, then $g\left(\alpha_{0} v\right) \in L^{1}(\Omega)$, so that $\alpha_{0} \mu_{F}+g\left(\alpha_{0} v\right)$ is a good measure. Applying Proposition 3 above, we conclude that $\alpha_{0} \mu_{F} \in \mathcal{G}(g)$.

We now present the

Proof of Theorem 2. Set $\ell_{k}=2^{-\frac{N}{N-2} k}, \forall k \geq 1$. We now fix an increasing sequence of positive integers $\left(k_{j}\right)$ such that

$$
\frac{g\left(j^{2}\right)}{2^{\frac{2 N}{N-2} k_{j}}} \leq \frac{1}{2^{j}} \quad \forall j \geq 1 .
$$

Let $F$ be the Cantor set associated to the subsequence $\left(\ell_{k_{j}}\right)$. We claim that $\alpha \mu_{F}$ is good for every $\alpha>0$.

In fact, since $2^{N k_{i}} \ell_{k_{i}}^{N-2}=1$ for every $i \geq 1$, we have

$$
\sum_{i=1}^{j+1} \frac{1}{2^{N k_{i}} \ell_{k_{i}}^{N-2}}=j+1 \leq 2 j \quad \forall x \in F_{j} \backslash F_{j+1} .
$$

Moreover,

$$
2^{N k_{j}} \ell_{k_{j}}^{N}=\frac{1}{2^{\frac{2 N}{N-2} k_{j}}} .
$$

Thus, for every $\beta>0$, we have

$$
\sum_{j=1}^{\infty} g\left(\beta \sum_{i=1}^{j+1} \frac{1}{2^{N k_{i}} \ell_{k_{i}}^{N-2}}\right) 2^{N k_{j}} \ell_{k_{j}}^{N} \leq \sum_{j=1}^{\infty} \frac{g(2 \beta j)}{2^{\frac{2 N}{N-2} k_{j}}} .
$$


Since $2 \beta j \leq j^{2}$ for $j \geq 1$ sufficiently large, it then follows from (4.9) that the right-hand side of (4.10) is finite for every $\beta>0$. Applying Proposition 4, we conclude that $\alpha \mu_{F}$ is a good measure for every $\alpha>0$.

On the other hand, since $2^{N k_{j}} \ell_{k_{j}}^{N-2}=1, \forall j \geq 1$, we deduce from Lemma 3 that $\mathcal{H}^{N-2}(F) \in(0, \infty)$. Thus, by $(4.2)$, we have

$$
\mu_{F}=\frac{1}{\mathcal{H}^{N-2}(F)} \mathcal{H}^{N-2}\left\lfloor_{F}\right.
$$

Therefore, $\alpha \mathcal{H}^{N-2}\left\lfloor_{F}\right.$ is good for every $\alpha>0$.

Proof of Theorem 3. Let $\left(k_{j}\right)$ be an increasing sequence of positive integers such that

$$
\frac{g\left(j^{3}\right)}{2^{\frac{2 N}{N-2} k_{j}}} \leq \frac{1}{2^{j}} \quad \forall j \geq 1
$$

Let

$$
\ell_{k}=\frac{1}{j^{\frac{1}{N-2}} 2^{\frac{N k}{N-2}}} \quad \text { if } k_{j-1}<k \leq k_{j}
$$

with the convention that $k_{0}=0$. Let $F$ be the Cantor set associated to the subsequence $\left(\ell_{k_{j}}\right)$. By Lemma 3 , we know that $\mathcal{H}^{N-2}(F)=0$. We now show that $\mu_{F}$ is a good measure relative to $g$.

Since $2^{N k_{i}} \ell_{k_{i}}^{N-2}=\frac{1}{i}$, we have

$$
\sum_{i=1}^{j+1} \frac{1}{2^{N k_{i}} \ell_{k_{i}}^{N-2}}=\frac{(j+1)(j+2)}{2} \leq 3 j^{2} .
$$

Moreover,

$$
2^{N k_{j}} \ell_{k_{j}}^{N}=\frac{1}{j^{\frac{N}{N-2}} 2^{\frac{2 N}{N-2} k_{j}}} \leq \frac{1}{2^{\frac{2 N}{N-2} k_{j}}} .
$$

Thus, for every $\beta>0$, we have

$$
\sum_{j=1}^{\infty} g\left(\beta \sum_{i=1}^{j+1} \frac{1}{2^{N k_{i}} \ell_{k_{i}}^{N-2}}\right) 2^{N k_{j}} \ell_{k_{j}}^{N} \leq \sum_{j=1}^{\infty} \frac{g\left(3 \beta j^{2}\right)}{2^{\frac{2 N}{N-2} k_{j}}} .
$$

Since $3 \beta j^{2} \leq j^{3}$ for $j \geq 1$ sufficiently large, it then follows from (4.11) that the right-hand side of (4.12) is finite for every $\beta>0$. Applying Proposition 4, we conclude that $\mu_{F}$ is a good measure. The proof of Theorem 3 is complete.

\section{Proof of Theorem 1}

When $N \geq 3$, Theorem 1 follows from Theorem 2 (or Theorem 3 ) and the following well-known 
Proposition 5 Let $K \subset \Omega$ be a compact set. If $\mathcal{H}^{N-2}(K)<\infty$, then cap $(K)=0$.

We refer the reader to e.g. [7] for a proof of Proposition 5.

We now deal with the case $N=2$. We shall need the following

Lemma 4 Assume $N=2$. Let $F \subset \Omega$ be the Cantor set associated to the subsequence $\left(\ell_{k_{j}}\right)$. Then,

$$
\operatorname{cap}(F)=0 \quad \text { if and only if } \quad \sum_{j=1}^{\infty} \frac{1}{4^{k_{j}}} \log \frac{1}{\ell_{k_{j}}}=\infty .
$$

When $F$ is a standard Cantor set, (5.1) is Carleson's test (see [7, p.31]) for determining whether $F$ has zero capacity. The same proof as in [7] can be used to establish Lemma 4. We present a different argument based on Proposition 2 above.

Proof of Lemma 4. $(\Leftarrow)$ Suppose

$$
\sum_{j=1}^{\infty} \frac{1}{4^{k_{j}}} \log \frac{1}{\ell_{k_{j}}}=\infty
$$

It then follows from Proposition 2 that $v=+\infty$ on $F$, where $v$ is the solution of (3.2). Since $v$ is superharmonic, we can apply Theorem 7.33 in [9] to conclude that $\operatorname{cap}(F)=0$.

$(\Rightarrow)$ Assume that

$$
\sum_{j=1}^{\infty} \frac{1}{4^{k_{j}}} \log \frac{1}{\ell_{k_{j}}}<\infty
$$

Let $v$ be the solution of (3.2). It follows from (5.2) and Proposition 2 that $v$ is uniformly bounded in $\Omega$. Thus, the measure $\mu_{F}$ is diffuse. Since $\mu_{F}$ is concentrated in $F$, we must have cap $(F)>0$. The proof of Lemma 4 is complete.

Remark 1 Here is the counterpart of (5.1) in dimension $N \geq 3$ :

$$
\operatorname{cap}(F)=0 \quad \text { if and only if } \quad \sum_{j=1}^{\infty} \frac{1}{2^{N k_{j}} \ell_{k_{j}}^{N-2}}=\infty .
$$

The proof of (5.3) follows along the same lines.

The analog of Proposition 4 in dimension $N=2$ is given by the next 
Proposition 6 Assume $N=2$. Let $F$ be the Cantor set associated to the subsequence $\left(\ell_{k_{j}}\right)$. There exists $C>0$ (depending on $\theta$ ) such that if

$$
\sum_{j=1}^{\infty} g\left(C \alpha_{0} \sum_{i=1}^{j+1} \frac{1}{4^{k_{i}}} \log \frac{1}{\ell_{k_{i}}}\right) 4^{k_{j}} \ell_{k_{j}}^{2}<\infty \quad \text { for some } \alpha_{0}>0,
$$

then $\alpha_{0} \mu_{F} \in \mathcal{G}(g)$.

The proof of Proposition 6 is based on Proposition 2 and shall be omitted.

We may now present the

Proof of Theorem 1 completed. Let $\ell_{k}=4^{-4^{k}}, \forall k \geq 1$. We now fix an increasing sequence of positive integers $\left(k_{j}\right)$ such that

$$
\frac{g\left(j^{2}\right)}{4^{4^{k_{j}}}} \leq \frac{1}{2^{j}} \quad \forall j \geq 1
$$

Let $F$ be the Cantor set associated to the subsequence $\left(\ell_{k_{j}}\right)$. Note that

$$
\frac{1}{4^{k_{i}}} \log \frac{1}{\ell_{k_{i}}}=\log 4 \quad \forall i \geq 1 .
$$

In particular,

$$
\sum_{i=1}^{j+1} \frac{1}{4^{k_{i}}} \log \frac{1}{\ell_{k_{i}}}=(j+1) \log 4 \leq 4 j .
$$

It then follows from Lemma 4 that cap $(F)=0$. We now show that $\mu_{F}$ is a good measure.

Since

$$
\left|F_{j} \backslash F_{j+1}\right| \leq\left|F_{j}\right|=4^{k_{j}} \ell_{k_{j}}^{2}=\frac{1}{4^{24^{k_{j}}-k_{j}}} \leq \frac{1}{4^{4^{k_{j}}}},
$$

then, for every $\beta>0$, we have

$$
\sum_{j=1}^{\infty} g\left(\beta \sum_{i=1}^{j+1} \frac{1}{4^{k_{i}}} \log \frac{1}{\ell_{k_{i}}}\right) 4^{k_{j}} \ell_{k_{j}}^{2} \leq \sum_{j=1}^{\infty} \frac{g(4 \beta j)}{4^{4^{k_{j}}}} .
$$

In view of (5.5), we conclude that the right-hand side of (5.6) is finite for every $\beta>0$. Thus, by Proposition 6 above, $\mu_{F}$ is good. The proof of Theorem 1 is complete.

\section{Acknowledgments}

The author is deeply grateful to H. Brezis for his encouragement; this work is dedicated to him with admiration and gratitude.

We warmly thank L. Orsina, I. Shafrir, and L. Véron for interesting discussions. This work was supported by the NSF grant DMS-0111298 and Sergio Serapioni, Honorary President of Società Trentina Lieviti-Trento (Italy). 


\section{References}

[1] D. Bartolucci, F. Leoni, L. Orsina, and A. C. Ponce, Semilinear equations with exponential nonlinearity and measure data. To appear in Ann. Inst. H. Poincaré Anal. Non Linéaire.

[2] Ph. Bénilan and H. Brezis, Nonlinear problems related to the Thomas-Fermi equation. J. Evol. Equ. 3 (2004), 673-770. Dedicated to Ph. Bénilan.

[3] H. Brezis, M. Marcus, and A. C. Ponce, Nonlinear elliptic equations with measures revisited. To appear in Annals of Math. Studies, Princeton University Press. Part of the results were announced in a note by the same authors: $A$ new concept of reduced measure for nonlinear elliptic equations, C. R. Acad. Sci. Paris, Ser. I 339 (2004), 169-174.

[4] H. Brezis and A. C. Ponce, Reduced measures on the boundary. To appear in J. Funct. Anal.

[5] H. Brezis and W. A. Strauss, Semilinear second-order elliptic equations in $L^{1}$. J. Math. Soc. Japan 25 (1973), 565-590.

[6] H. Brezis and L. Véron, Removable singularities for some nonlinear elliptic equations. Arch. Rational Mech. Anal. 75 (1980/81), 1-6.

[7] L. Carleson, Selected problems on exceptional sets. Van Nostrand Mathematical Studies, No. 13, Van Nostrand, Princeton, 1967.

[8] L. Dupaigne, A. C. Ponce, and A. Porretta, Elliptic equations with vertical asymptotes in the nonlinear term. In preparation.

[9] L. L. Helms, Introduction to potential theory. Pure and Applied Mathematics, vol. XXII, Wiley-Interscience, New York, 1969.

[10] M. Marcus and L. Véron. Nonlinear capacities associated to semilinear elliptic equations. In preparation.

[11] P. Mattila, Geometry of sets and measures in Euclidean spaces. Cambridge Studies in Advanced Mathematics, vol. 44, Cambridge University Press, Cambridge, 1995.

[12] J. L. Vázquez, On a semilinear equation in $\mathbb{R}^{2}$ involving bounded measures. Proc. Roy. Soc. Edinburgh Sect. A 95 (1983), 181-202.

A. C. Ponce

Institute for Advanced Study

Princeton, NJ 08540

USA

e-mail: augponce@math.ias.edu 